\title{
ET-Hollow Module and ET-Lifting Module
}

\author{
Firas sh. Fandi ${ }^{1}$, \\ ${ }^{1}$ Mathematics Department College of Education for Pure \\ Sciences, \\ University Of Anbar, Ramadi, Iraq \\ Frisshker1978@gmail.com
}

\author{
Sahira M. Yaseen ${ }^{2}$ \\ ${ }^{2}$ Mathematics Department College of Science \\ University of Baghdad, Iraq \\ Sahira.mahmood@gmail.com
}

\begin{abstract}
Let $\mathrm{M}$ be a R-module, where $\mathrm{R}$ be a commutative ring with identity,
In this paper, we defined a new types of module namely "ET-hollow(ET-holl.) and ET-lifting(ET-lift.) modules". An R-module M is called ETholl. module, if for all sub-module $\mathrm{H}$ of $\mathrm{M}$ then $\mathrm{H} \ll_{E T} \mathrm{M}$. An R-module $\mathrm{M}$ is called An R-module $\mathrm{M}$ is called $\mathrm{ET}$-lifting module, if for all $\mathrm{H} \leq \mathrm{M}$, there exists $\mathrm{X} \leq_{\oplus} \mathrm{M}$ and $\mathrm{L} \ll_{E T} \mathrm{M}$, such that $\mathrm{H}=\mathrm{X}+\mathrm{L}$. We give many characterizations of $\mathrm{ET}$-holl. and $\mathrm{ET}$-lifting modules, Also we give the relation between T-hollow and ET-holl. and relation between T-lifting modules and ET-lift. modules.
\end{abstract}

Key Words: T-small sub-module, ET-small sub-module, e-hollow module, T-hollow module,ET-hollow module, T-lifting module, ET-lifting module

$* * * * *$

\section{Introduction}

Throughout this paper, $\mathrm{M}$ be an $\mathrm{R}$-module; where $\mathrm{R}$ be any a commutative ring and $\mathrm{R}$ with unity, a proper sub-module $H$ of $M$ is called small $(H \ll M)$, if for all $X \leq M$, such that $M=H+X$ then $M=X[1]$. A sub-module $H$ of $M$ is called essential of $M$, if $H \cap B=0, \forall B \leq M$, then $B=0$ [2]. A sub-module $H$ of $M$ is e-small $\left(H \ll_{e} M\right)$, if for every submodule $\mathrm{B}$ of $\mathrm{M}$ such that $\mathrm{H}+\mathrm{B}=\mathrm{M}$, then $\mathrm{B}=\mathrm{M}[3]$. Let $\mathrm{T} \leq M, \mathrm{~A}$ sub-module $\mathrm{H}$ of $\mathrm{M}$ is said to be "T-small sub-module of $M^{\prime \prime}$, if for all $K \leq M$ such that $T \subseteq H+K$, then $T \subseteq K$ [4]. Recalled a module $M$ is e-hollow module, if for all proper sub-module $H$ of $M$ is e-small of $M[5]$. $M$ is e-lifting module if for all sub-module $H$ of $M$, there exists $K \leq M$ such that $\frac{H}{K} \ll_{e} \frac{M}{K}[6]$. In[7] we defined ET-small sub-module of $\mathrm{M}$, Let $\mathrm{T} \leq \mathrm{M}$ and $\mathrm{A}$ sub-module $\mathrm{H}$ of $\mathrm{M}$ is "ET-small submodule of $\mathrm{M}^{\prime \prime}$, if for all $\mathrm{K} \leq_{e} \mathrm{M}$ such that $\mathrm{T} \subseteq \mathrm{H}+\mathrm{K}$, then $\mathrm{T} \subseteq \mathrm{K}$, and we give some properties of $\mathrm{ET}$-small sub-module of $\mathrm{M}$, Clearly. Every T-small sub-module of $\mathrm{M}$ is $\mathrm{ET}$-small sub-module of $\mathrm{M}$ but the converse is not true. Let $\mathrm{M}$ be an R-module and Let $T, N_{1}$ and $N_{2} \leq M$, Then $N_{1} \ll_{E T} M$ and $N_{2} \ll_{E T} M$ if and only if $N_{1}+N_{2} \ll \ll_{E T} M$. assume that $T, H$ and $L$ are sub-modules of any R-module $\mathrm{M}$ such that $\mathrm{T} \leq \mathrm{N}$ and $\mathrm{H} \leq \mathrm{L} \leq \mathrm{M}$ and $\mathrm{L} \ll_{\mathrm{e}} \mathrm{M}$, if $\mathrm{H} \ll_{E T} M$ then $\mathrm{H} \ll_{E T} \mathrm{~L}$, and there are many of other properties of ET-small [7].

In this work, we give the concept of ET-holl. module and ET-lifting module. An module M is called ET-holl. module, if $\forall \mathrm{H} \leq \mathrm{M}$ then $\mathrm{H} \ll_{E T} \mathrm{M}$. An R-module $\mathrm{M}$ is called ET-lifting module, if for all $\mathrm{H} \leq \mathrm{M}$, there exists $\mathrm{X}$ $\leq_{\oplus} \mathrm{M}$ and $\mathrm{L} \ll_{E T} \mathrm{M}$ such that $\mathrm{H}=\mathrm{X}+\mathrm{L}$. Also We give many properties of ET-holl. module and ET-lifting module.

In section 1, we defined ET-holl. module with we give some properties of ET-holl. module. Every T-hollow module is ET-holl. module, but the converse is not true, but if $\mathrm{M}$ be a non-zero module and $\mathrm{T}=\mathrm{M}$. thus $\mathrm{M}$ is $\mathrm{ET}$-holl. module iff $\mathrm{M}$ is e-holl. module. Let $\mathrm{T} \leq M$. If $\mathrm{M}$ is $\mathrm{ET}$-holl. module, then every essential sub-module $\mathrm{K}$ of $\mathrm{M}$ such that $T \subseteq K$, then $K$ is a $T$-hollow module. Let $(T \neq 0)$ be an essential sub-module of a module $M$, if $M$ is $E T$-holl. module, then $\mathrm{T}$ is indecomposable. there are some of other properties of ET-holl. module.

In section 2, we defined ET-lifting module with we give some properties of ET-lifting module. Also, we list many of their important properties. Also we give the relation between T-hollow and ET-holl. and relation between T-lifting modules and ET-lifting modules. 


\section{Section 1: ET-hollow module}

In section this, we defined ET-holl. module and we give many properties of ET-holl. module.

Definition 1.1:Let $T$ be a sub-module of $(M \neq 0)$ module. We say that $M$ is a $E T$-holl. module if for every sub-module $\mathrm{H}$ of $\mathrm{M}$ is a ET-small sub-module of $\mathrm{M}$.

\section{Remark and Example 1.2:}

1. Consider $Z_{6}$ as $Z$-module .Let $T=\{\underline{0}, \underline{3}\}$, then $Z_{6}$ is $E T$-holl. since the only essential sub-module of $Z_{6}$. Then the submodules $\mathrm{H}=\{0\},\{0,3\},\{0,2,4\}$ and $Z_{6}$, then $T \subseteq H+Z_{6}$. Implies that $T \subseteq Z_{6}$, then for every sub-module $H$ of $Z_{6}$ is a ETsmall sub-module of $\mathrm{Z}_{6}$.

2. Every $(M \neq 0) T$-hollow module is $E T$-holl. module, but the converse is not true, for example Consider $Z_{4}$ as $Z$ module. let $T=\{\underline{0}, \underline{2}\}$ and $B=\{\underline{0}, \underline{2}\}$, then $B$ is not $T$ - small sub-module of $Z_{4}$, since $T \subseteq B+\{0\}$, but $T \nsubseteq\{0\}$. So $Z_{4}$ is not $T$ hollow module . but $Z_{4}$ is $E T$-holl. module. Since for every sub-module $H$ of $M$ is a ET-small sub-module of $M$, since $H$ $=\{0\},\{0,2\}$ and $Z_{4}$ are sub-modules of $Z_{4}$ then $T \subseteq H+X, \forall X \leq_{e} Z_{4}$, but the only essential sub-modules of $Z_{4}$ are $\{0,2\}$ and $Z_{4}$ since $T \subseteq\{0,2\}$ and $T \subseteq Z_{4}$. So every sub-module $H$ of $Z_{4}$ is $E T$-small sub-module of $Z_{4}$. Then $Z_{4}$ is $E T$-hollow module.

3. Let $(M \neq 0)$ be an uniform module and let $T=M$, so $M$ is holl. module iff $M$ is ET-holl. module.

4. Let $(M \neq 0)$ be an module and $T=M$. so $M$ is ET-holl. module iff $M$ is e-holl. module.

5. Let $(M \neq 0)$ be an module and $T=0$, hence $M$ is ET-holl. module. by remark (1.2-3) [7].

Proof 3: Assume that $(M \neq 0)$ be an uniform module such that $T=M$ and $M$ is hollow module to prove $M$ is ETholl. module . suppose that $H \leq M$ to prove $H \ll_{E T} M$, let $T \subseteq H+X$, for every essential sub-module $X$ of $M$, to prove $T \subseteq X$, since $T=M$ so $M \subseteq H+X$ and $H+X \subseteq M$ therefor $M=H+X$, but $M$ is hollow module then $H \ll M$, so $M=X$ implies that $T=X$ therefor $T \subseteq X$ then $H \ll_{E T} M$. thus $M$ is $E T$-holl. module. Conversely let $M$ is ET-holl. module, to show $M$ is $E T$-holl. module. let $H \leq M$, to show $H \ll M$, so $M=H+X$ then $T \subseteq H+X, \forall X \leq M$, but $M$ is uniform module then $X \leq_{e} M$, since $M$ is ET-holl. module, then $H \ll_{E T} M$, therefor $T \subseteq X$ then $M \subseteq X$ and $X \subseteq M$, thus $\mathrm{M}=\mathrm{X}$, implies that $\mathrm{H} \ll M$, then $\mathrm{M}$ is hollow module.

4. Let $M$ is hollow module and $T=M$ and $M$ is hollow module to prove $M$ is e-hollow module. let $H \leq M$ to show $H \ll{ }_{e} M$, let $M=H+B$, for every essential sub-module $B$ of $M$, to prove $B=M$, since $T=M$ so $T=H+B$ therefor $T$ $\subseteq \mathrm{H}+\mathrm{B}$, but $\mathrm{M}$ is $\mathrm{ET}$-holl. module, then $\mathrm{H} \ll_{E T} \mathrm{M}$, so $T \subseteq B$ thus $\mathrm{M} \subseteq \mathrm{B}$ and $\mathrm{B} \subseteq \mathrm{M}$ then $\mathrm{B}=\mathrm{M}$, hence $\mathrm{H} \ll_{e} \mathrm{M}$. then $\mathrm{M}$ is e-hollow module. Conversely let $M$ is e-hollow module, to show $M$ is $E T$-hollow module . let $H \leq M$, to prove $H$ $\ll_{E T} \mathrm{M}$, let $T \subseteq H+A, \forall A \leq{ }_{e} M$, since $T=M$ so $M \subseteq H+A$ and $H+A \subseteq M$ thus $M=H+A$, since $M$ is e-hollow module then $\mathrm{H} \ll{ }_{e} \mathrm{M}$, therefor $\mathrm{A}=\mathrm{M}$, then $\mathrm{T}=\mathrm{A}$ therefor $\mathrm{T} \subseteq \mathrm{A}$, thus $\mathrm{H} \ll_{E T} \mathrm{M}$ then $\mathrm{M}$ is ET-hollow module.

Proposition 1.3: Let $T$ be sub-module of a non -zero module $M$.If $M$ is ET-holl. module, then every essential submodule $\mathrm{H}$ of $\mathrm{M}$ such that $\mathrm{T} \subseteq \mathrm{H}$, then $\mathrm{H}$ is a $\mathrm{T}$-hollow module.

Proof:- Let $\mathrm{H}$ be an essential sub-module of $\mathrm{M}$ such that $\mathrm{T} \subseteq \mathrm{H}$. to prove $\mathrm{H}$ is a $\mathrm{ET}$-holl. module. so Let $\mathrm{L}$ be a submodule of $H$, show to $L \ll_{E T} H$, Since $H \leq M$ and $M$ be an ET-holl. module, then $L \ll_{E T} M$. Then, by (1.4) [7]. then $\mathrm{L} \ll_{E T} \mathrm{H}$. Thus $\mathrm{H}$ is a T-hollow module.

Proposition 1.4: Let $T$ be a non-zero essential sub-module of a module $M$.If $M$ is ET-holl. module .Then $T$ is indecomposable.

Proof:- Let $\mathrm{M}$ be an ET-holl. module and $\mathrm{T}$ is decomposable, then $\mathrm{T}=\mathrm{H} \oplus \mathrm{X}$, for some $\mathrm{H}$ and $\mathrm{X}$ are non-trivial submodules of $\mathrm{T}$. Therefore $\mathrm{T} \nsubseteq \mathrm{H}$. since $\mathrm{X} \leq \mathrm{T}=(\mathrm{H} \oplus \mathrm{X}) \leq_{e} \mathrm{M}$, then $\mathrm{X} \leq_{e} \mathrm{M}$, since $\mathrm{M}$ is ET-holl. module and $\mathrm{T} \subseteq \subseteq \mathrm{H} \oplus \mathrm{X}$, then $\mathrm{H} \ll \ll_{E T} \mathrm{M}$. therefore $\mathrm{T} \subseteq \mathrm{X}$ and $\mathrm{X} \subseteq \mathrm{T}$, hence $\mathrm{T}=\mathrm{X}$ which is a contradiction. Then $\mathrm{T}$ is indecomposable .

Proposition 1.5: let $f: M \rightarrow N$ be an epimorphism, where $M$ and $N$ are a non -zero modules, if $M$ be an ET-holl. module, Then $\mathrm{N}$ is $\mathrm{Ef}(\mathrm{T})$-hollow module .

Proof:- Let $\mathrm{M}$ is an ET-holl. module and let $f: \mathrm{M} \rightarrow \mathrm{N}$ be an epimorphism . To prove $\mathrm{N}$ is $\mathrm{Ef}$ (T)-hollow module. Let $\mathrm{H}$ be a sub-module of $\mathrm{N}$, to prove $\mathrm{H} \ll \ll_{E f(T)} \mathrm{N}$, since $f$ be an epimorphism, there exist $\mathrm{K} \leq \mathrm{M}$ such that $\mathrm{f}(\mathrm{K})=\mathrm{H}$, But $\mathrm{M}$ 
is ET-holl. module, then $\mathrm{K} \ll_{E T} \mathrm{M}$, and by lemma (1.8)[7], we get $\mathrm{f}(\mathrm{k}) \ll_{E f(T)} \mathrm{N}$, implies that $\mathrm{H} \ll_{E f(T)} \mathrm{N}$. then $\mathrm{N}$ is Ef(T)-hollow module.

Proposition 1.6: Let $\mathrm{T}$ and $\mathrm{H}$ be sub-modules of a module $\mathrm{M}$ such that $\mathrm{H} \subseteq \mathrm{T} . \mathrm{H} \leq_{c} \mathrm{M}$. If $\mathrm{H} \ll_{E T} \mathrm{M}, \frac{M}{H}$ be any $\mathrm{E}\left(\frac{T}{H}\right)$ - holl. module, hence $\mathrm{M}$ is ET-holl.

Proof:- Let $\mathrm{H} \ll_{E T} \mathrm{M}$ and $\frac{M}{H}$ is $\mathrm{E}\left(\frac{T}{H}\right)$-holl. module. To prove $\mathrm{M}$ is $\mathrm{ET}$-holl. let $\mathrm{B}$ be a sub-module of $\mathrm{M}$, To prove $\mathrm{B} \ll<_{E T} \mathrm{M}$, let $\mathrm{T} \subseteq \mathrm{B}+\mathrm{L}, \forall \mathrm{L} \leq_{e} \mathrm{M}$. Then $\frac{T}{H} \subseteq \frac{B+L}{H}$ and hence $\frac{T}{H} \subseteq \frac{B+H}{H}+\frac{L+H}{H}$. since $\left(\mathrm{L} \leq \mathrm{L}+\mathrm{H} \leq \mathrm{M}\right.$ and $\mathrm{L} \leq_{e} \mathrm{M}$ then $\mathrm{L}+\mathrm{H} \leq_{e} \mathrm{M}$ and since $\mathrm{H} \leq_{c} \mathrm{M}$ then $\left.\frac{L+H}{H} \leq_{e} \frac{M}{H}\right)$, but $\frac{M}{H}$ is $\mathrm{E}\left(\frac{T}{H}\right)$-hollow module, therefore $\frac{B+H}{H} \ll_{E}\left(\frac{T}{H}\right) \frac{M}{H}$, hence $\frac{T}{H} \subseteq$ $\frac{L+H}{H}$, Thus $\mathrm{T} \subseteq \mathrm{L}+\mathrm{H}$. Since $\mathrm{H} \ll_{E T} \mathrm{M}$, then $\mathrm{T} \subseteq \mathrm{L}$. hence $\mathrm{M}$ is $\mathrm{ET}$-holl. module.

Proposition 1.7: Let $M$ be a module and $H$ and $T$ are sub-modules of $M$, such that $H \Varangle T$ and $H \leq_{e} M$. If $M$ is ET-holl. Module, and $\frac{T}{H}$ is non-zero finitely generated; hence $\mathrm{T}$ be a finitely generated.

Proof: Let $\frac{T}{H}=R\left(x_{1}+\mathrm{H}\right)+R\left(x_{2}+\mathrm{H}\right)+\ldots+R\left(x_{n}+\mathrm{H}\right)=\left(R x_{1}+\mathrm{H}\right)+\left(R x_{2}+\mathrm{H}\right)+\ldots+\left(R x_{n}+\mathrm{H}\right)$ where $x_{i} \in \mathrm{T}$, for all $i=1, \ldots, n$. then $R x_{1}+$ $R x_{2}+\ldots+R x_{n} \subseteq \mathrm{T}$.To prove $\mathrm{T} \subseteq R x_{1}+R x_{2}+\ldots+R x_{n}$, let $a \in \mathrm{T}$. Then $a+\mathrm{H} \in \frac{T}{H}$, then there exist $r_{1}, \ldots, r_{n} \in \mathrm{R}$, such that $\mathrm{a}+\mathrm{H}=r_{1}$ $\left(x_{1}+\mathrm{H}\right)+r_{2}\left(x_{2}+\mathrm{H}\right)+\ldots+r_{n}\left(x_{n}+\mathrm{H}\right)=\left(r_{1} x_{1}+r_{2} x_{2}+\ldots+r_{n} x_{n}\right)+\mathrm{H}$. Hence $\mathrm{a}=\left(r_{1} x_{1}+r_{2} x_{2}+\ldots+r_{n} x_{n}\right)+h$, for some $h \in \mathrm{H}$ . Therefore $\mathrm{T}=\left(R x_{1}+R x_{2}+\ldots+R x_{n}\right)+\mathrm{H}$. Since $\mathrm{H} \lessgtr \mathrm{T}$, since $\mathrm{M}$ is $\mathrm{ET}$-holl. module, so $\mathrm{H} \ll E T$ M. So $\mathrm{T} \subseteq R x_{1}+R x_{2}+\ldots+$ $R x_{n}$. hence $T=\left(R x_{1}+R x_{2}+\ldots+R x_{n}\right)$.

\section{Section 2: ET-lifting module}

In section this, we defined ET-lift. module with we give many properties of ET-lifting module.

Definition 2.1:- suppose $\mathrm{T}$ is sub-module of a module $\mathrm{M}$. $\mathrm{M}$ is said to be a $\mathrm{ET}$-lifting module, if every sub-module $\mathrm{H}$ of $M$, there exists a direct summand $K$ of $M$ and $L \ll_{E T} M$ such that $H=K+L$.

\section{Remark and Example 2.2:}

1. $Z_{4}$ as $Z$-module is $E\left(Z_{4}\right)$-lifting module.

2. Let $M$ be a module and $T=M$.Then $M$ is $E T$-lifting module if and only if $M$ is e-lifting module.

Proof:- Let $T=M$ and $M$ is $E(T)$-lifting module, to prove $M$ is e-lifting module. Let $H$ be sub-module of $M$, hence there exist $B \leq_{\oplus} M$ and $\mathrm{A} \ll_{E T} \mathrm{M}$ such that $\mathrm{H}=\mathrm{B}+\mathrm{A}$. By the modular law $\mathrm{H}=\mathrm{H} \cap \mathrm{M}=\mathrm{H} \cap\left(\mathrm{B} \oplus \mathrm{B}^{\prime}\right)=\mathrm{B} \oplus\left(\mathrm{H} \cap \mathrm{B}^{\prime}\right)$. Take $\mathrm{A}=\mathrm{H} \cap \mathrm{B}^{\prime}$ and hence $A \ll_{e} M$, by [(1.2-4)[7].Thus $M$ is e-lift. module. For the converse, suppose that $M$ is $E T$-lifting module and let $H$ be sub-module of $M$, then $H=B \oplus L$, where $B \leq_{\oplus} M$ and $A \ll_{e} M$. by [(1.2-4) )[7]. hence $H \ll \ll_{E T} M$. then $M$ is $E(T)$ lift. module.

3. every T-lifting module is ET-lift. module. But the converse is not true, for the example consider $Z_{8}$ as Z-module and let $\mathrm{T}=\{\underline{0}, \underline{4}\}$ then $Z_{8}$ is not $\mathrm{T}$-lifting module, To prove that , suppose that is not and let $\mathrm{H}=\{\underline{0}, \underline{4}\}$, then there exists $\mathrm{K} \leq_{\oplus} Z_{8}$ and $\mathrm{L} \ll_{T} Z_{8}$ such that $\mathrm{H}=\mathrm{K}+\mathrm{L}$. Since $Z_{8}$ indecomposable, then $\mathrm{K}=0$ and $\mathrm{H}=\{\underline{0}, \underline{4}\}$. But $\mathrm{H}=\mathrm{L}=\{\underline{0}, \underline{4}\}$ is not $\mathrm{T}$-small sub-module in $Z_{8}$.implies that $Z_{8}$ is not T-lifting module. But $\mathrm{H}=\mathrm{L}$ is ET-small sub-module in $Z_{8}$ [7], then $Z_{8}$ is an ETlifting module.

Remark 2.3: assume that $M$ be a semisimple module. So $M$ is ET-lifting module, for all sub-module $T$ of $M$.

Proof:- Let $M$ is an semisimple module and $H$ be sub-module of $M$. Thus $H=H+0$, where $H \leq \leq_{\oplus} M$ and $0 \ll{ }_{E T} M$ .Then $\mathrm{M}$ is ET-lifting module.

Remark 2.4: Let $M$ be an ET-lifting module. Then for all sub-module $B$ of $M$ such that $T \subseteq B$ is also $E T$-lifting.

Proof:- let $M$ is $E T$-lifting and $B$ be a sub-module of $M$ such that $T \subseteq B$. To prove $B$ is $E T$-lifting, let $H$ is a sub-module of $\mathrm{B}$.thus $H \subseteq M$ and since $\mathrm{M}$ is $\mathrm{ET}$-lifting, then $\mathrm{H}=\mathrm{K}+\mathrm{L}$, where $\mathrm{K} \leq_{\oplus} \mathrm{M}$ and $\mathrm{L} \ll_{E T} \mathrm{M}$ and since $\mathrm{K} \leq \mathrm{B} \leq \mathrm{M}$ and $\mathrm{K} \leq_{\oplus} \mathrm{M}$ then $K \leq_{\oplus} B$ [2]. Since $T \subseteq B$, then $L \ll_{E T} B$, by (1.4)[7].Thus B is ET-lifting . 
Recall that a sub-module $H$ of a module $M$ is called ((projective invariant)), if for every $P=P^{2} \in E$ En $(M), P(H) \leq H$, see [8].

Proposition 2.5: Let $T$ be a submodule of a module $M$.Consider the following statements:

1. For each submodule $X$ of $M$, there exists a decomposition $M=K \oplus K$ s such that $K \subseteq H$ and $\left(H \cap K K^{\prime}\right) \ll_{E T} M$.

2. $M$ is ET-lifting module.

Then $\mathbf{1}=\mathbf{2}$

$\mathbf{2}=\mathbf{2}$ If for all ET-small sub-module of $\mathrm{M}$ is projective invariant.

Proof: $(\mathbf{1 = 2})$ Let $\mathrm{H}$ be a sub-module of $\mathrm{M}$, there exists a decomposition $\mathrm{M}=\mathrm{K} \oplus \mathrm{K}^{\prime}$ such that $\mathrm{K} \subseteq \mathrm{H}$ and $\left(\mathrm{H} \cap \mathrm{K}^{\prime}\right) \ll_{E T} \mathrm{M}$. Now $\mathrm{H}=\mathrm{H} \cap \mathrm{M}=\mathrm{H} \cap\left(\mathrm{K} \oplus \mathrm{K}^{\prime}\right)=\mathrm{K} \oplus\left(\mathrm{H} \cap \mathrm{K}^{\prime}\right)$, by the Modular Law and let $\mathrm{L}=\left(\mathrm{H} \cap \mathrm{K}^{\prime}\right)$, then $\mathrm{H}=\mathrm{K}+\mathrm{L}$. Thus $\mathrm{M}$ is $\mathrm{ET}$-lifting module.

(2=) Let $M$ is ET-lifting module and for every ET-small sub-module of $M$ is (projective invariant) . Let $H$ be submodule of $\mathrm{M}$, Then $\mathrm{H}=\mathrm{K}+\mathrm{L}$, where $\mathrm{K} \leq_{\oplus} \mathrm{M}$ and $\mathrm{L} \ll_{E T} \mathrm{M}$. to prove $\mathrm{H} \cap \mathrm{K}^{\prime} \ll_{E T} \mathrm{M} . \mathrm{H}=\mathrm{H} \cap \mathrm{M}=\mathrm{H} \cap\left(K \oplus \mathrm{K}^{\prime}\right)=\mathrm{K} \oplus\left(\mathrm{H} \cap \mathrm{K}^{\prime}\right)$, by the Modular Law . Let $P: M \rightarrow K^{\prime}$ be the projection map. $P(L)=P(K+L)=P(H)=P\left(K \oplus\left(H \cap K^{\prime}\right)\right)=P\left(H \cap K^{\prime}\right)=H \cap K^{\prime}$. Since $L$ is ET-small in $M$, then by our assumption $L$ is projective invariant. So $P(L)=H \cap K^{\prime} \leq L$. Thus $H \cap K^{\wedge} \ll_{E T} M$, by (1.8) [7 ].

Theorem 2.6: Let $T$ be sub-module of a module $M$. Then the following statements are equivalent:

1. For all sub-module $\mathrm{H}$ of $\mathrm{M}$, there exists a decomposition $\mathrm{M}=\mathrm{K} \oplus \mathrm{K}^{\prime}, \mathrm{K} \subseteq \mathrm{H}$ and $\mathrm{H} \cap \mathrm{K}^{\prime} \ll_{E T} \mathrm{M}$.

2. For all sub-module $\mathrm{H}$ of $\mathrm{M}$, there exists $\beta \in E$ nd $(\mathrm{M})$ such that $\beta^{2}=\beta, \beta(\mathrm{M}) \subseteq \mathrm{H}$ and $(1-\beta)(\mathrm{H}) \ll_{E T} \mathrm{M}$.

Proof: $(\mathbf{1 = 2})$ Let $\mathrm{H}$ be sub-module of $\mathrm{M}$. Then there exists a decomposition $\mathrm{M}=\mathrm{K} \oplus \mathrm{K}^{\prime}$ such that $\mathrm{K} \subseteq \mathrm{H}$ and $\mathrm{H} \cap \mathrm{K}^{\prime} \ll_{E T} \mathrm{M}$. Let $\beta: M \rightarrow K$ be the projection map. Clearly that $\beta^{2}=\beta$ and $\mathrm{M}=\mathrm{K} \oplus \mathrm{K}^{\prime}=\beta(\mathrm{M}) \oplus(1-\beta(\mathrm{M})$, since $\alpha$ is onto then $\beta(\mathrm{M})=\mathrm{K} \subseteq \mathrm{H}$. Now $(1-\beta)(\mathrm{H})=\mathrm{H} \cap(1-\beta)(\mathrm{M})=\mathrm{H} \cap \mathrm{K}^{\prime}$ but $\mathrm{H} \cap \mathrm{K}^{\prime} \ll_{E T} \mathrm{M}$. then $(1-\beta)(\mathrm{H}) \ll_{E T} \mathrm{M}$.

$(\mathbf{2}=\mathbf{1})$ Let $\mathrm{H}$ be sub-module of $\mathrm{M}$. Then there exists $\beta \in E n d(\mathrm{M})$ such that $\left.\beta^{2}=\beta, \beta \mathrm{M}\right) \subseteq \mathrm{H}$ and $(1-\beta)(\mathrm{H}) \ll_{E T} \mathrm{M}$. Clearly that $\mathrm{M}=\beta(\mathrm{M}) \oplus(1-\beta)(\mathrm{M})$, then $\mathrm{M}=\left(\mathrm{K} \oplus \mathrm{K}^{\prime}\right)$. Let $\mathrm{D}=\beta(\mathrm{M})$ and $\mathrm{D}^{\prime}=(1-\beta)$ (M) . Then $\mathrm{H} \cap \mathrm{K}^{\prime}=\mathrm{H} \cap(1-\beta)(\mathrm{M})$. To prove $\mathrm{H} \cap(1-$ $\beta)(\mathrm{M})=(1-\beta)(\mathrm{H})$, let $\mathrm{a}=(1-\beta)(\mathrm{b}) \in(\mathrm{H} \cap(1-\beta)(\mathrm{M}))$. Since $(1-\beta)^{2}=(1-\beta)$, then $\left.\mathrm{a}=(1-\beta)^{2}(\mathrm{~b})=(1-\beta)(1-\beta)(\mathrm{b})\right)=(1-\beta)(\mathrm{a}) \in(1-\beta)$ $(\mathrm{H})$. Now let $\mathrm{a}=(1-\beta)(\mathrm{b}) \in(1-\beta)(\mathrm{H}), \mathrm{b} \in \mathrm{H}$, then $\mathrm{a} \in(1-\beta)(\mathrm{M})$. $\mathrm{a}=(1-\beta)(\mathrm{b})=\mathrm{b}-\beta(\mathrm{b}) \in \mathrm{H}$. Thus $\mathrm{a} \in \mathrm{H} \cap(1-\beta)(\mathrm{M})$.

Now $(1-\beta)(\mathrm{H})=\mathrm{H} \cap(1-\beta)(\mathrm{M})=\mathrm{H} \cap K^{\prime}$, But $(1-\beta)(\mathrm{X}) \ll_{E T} \mathrm{M}$, then $\mathrm{H} \cap \mathrm{K}^{`} \ll_{E T} \mathrm{M}$

Proposition 2.7: let $\mathrm{M}$ be ET-lifting module and let $\mathrm{B}$ be a sub-module of $\mathrm{M}$ such that for every direct summand $\mathrm{K}$ of $\mathrm{M}, \frac{K+B}{B}$ is direct summand of $\frac{M}{B}$, then $\frac{M}{B}$ is $\mathrm{E}\left(\frac{T+B}{B}\right)$-lifting module.

Proof:- Let $\frac{H}{B}$ is a sub-module of $\frac{M}{B}$. but $\mathrm{M}$ is $\mathrm{ET}$-lifting, so $\mathrm{H}=\mathrm{K}+\mathrm{L}, \forall \mathrm{K} \leq{ }_{\oplus} \mathrm{M}$ and $\mathrm{L} \ll_{E T} \mathrm{M}$. Hence $\frac{H}{B}=\frac{K+L}{B}=\frac{K+B}{B}+\frac{L+B}{B}$ .By our assumption, $\frac{K+B}{B} \leq_{\oplus} \frac{M}{B}$.To prove $\frac{L+B}{B} \ll_{E\left(\frac{T+B}{B}\right)} \frac{M}{B}$. Let $\frac{Z}{B} \leq_{e} \frac{M}{B}$ such that $\frac{T+B}{B} \subseteq \frac{H+B}{B}+\frac{Z}{B}=\frac{H+B+Z}{B}$, then $\mathrm{T} \subseteq \mathrm{T}+\mathrm{B} \subseteq \mathrm{H}+(\mathrm{B}+\mathrm{Z})$. (Since $\frac{Z}{B} \leq_{e} \frac{M}{B}$ then $\mathrm{Z} \leq_{e} \mathrm{M}$ and $\mathrm{Z} \leq \mathrm{B}+\mathrm{Z} \leq \mathrm{M}$, then $\left.\mathrm{B}+\mathrm{Z} \leq_{e} \mathrm{M}\right)$. Since $\mathrm{L} \ll_{E T} \mathrm{M}$, then $\mathrm{T} \subseteq \mathrm{B}+\mathrm{Z}$ and hence $\frac{T+B}{B} \subseteq \frac{Z+B}{B}=\frac{Z}{B}$. Thus $\frac{M}{B}$ is $\mathrm{E}\left(\frac{T+B}{B}\right)$-lifting module.

Recall that $M$ is called a (distributive module) if for every sub-modules $A, B$ and $C$ of $M, A+(B \cap C)=(A+B) \cap(A+C)$ and $A \cap(B+C)=(A \cap B)+(A \cap C)$, see[9].

Corollary2.8: suppose that $M$ is $E T$-lifting, $M$ is distributive module and $\mathrm{A}$ be a sub-module of $\mathrm{M}$.Then $\frac{M}{B}$ is $\frac{T+B}{B}$-lifting module.

Proof: Let $\mathrm{K}$ be a direct summand of $\mathrm{M}$. Then $\mathrm{M}=\mathrm{K} \oplus \mathrm{K}^{\prime}$, for some sub-module $\mathrm{K}^{\wedge}$ of $\mathrm{M}$. Hence $\frac{M}{B}=\frac{K+K^{\prime}}{B}=\frac{K+B}{B}+\frac{K^{\prime}+B}{B}$ . Since $\mathrm{M}$ is distributive, then $(\mathrm{K}+\mathrm{B}) \cap\left(\mathrm{K}^{\prime}+\mathrm{B}\right)=\left((\mathrm{K}+\mathrm{B}) \cap \mathrm{K}^{\prime}\right)+((\mathrm{K}+\mathrm{B}) \cap \mathrm{B})=\left(\mathrm{K}^{\prime} \mathrm{K}^{\prime}\right)+\left(\mathrm{B} \cap \mathrm{K}^{\prime}\right)+(\mathrm{K} \cap \mathrm{B})+\mathrm{B}=\mathrm{B}$. Hence $\frac{M}{B}=\frac{K+B}{B} \oplus$ $\frac{K^{`}+B}{B}$.Thus, by (2.8), then $\frac{M}{B}$ is $\mathrm{E}\left(\frac{T+B}{B}\right)$-lifting module.

Proposition2.9: Let $\mathrm{M}=\mathrm{M}_{1} \oplus \mathrm{M}_{2}$ be a module such that $\mathrm{R}=A_{n n}\left(\mathrm{M}_{1}\right)+A_{n n}\left(\mathrm{M}_{2}\right)$. If $\mathrm{M}_{1}$ is $E T_{1}$-lifting and $\mathrm{M}_{2}$ is $E T_{2}$-lifting, then $M=M_{1} \oplus M_{2}$ is $E\left(T_{1} \oplus T_{2}\right)$-lifting module.

Proof:- Let $H$ be sub-module of a module $M$. Since $R=A_{n n}\left(M_{1}\right)+A_{n n}\left(M_{2}\right)$, then then $H=H_{1} \oplus H_{2}$, where $H_{1} \leq M_{1}$ and $H_{2} \leq$ $M_{2}$ [10] . But $M_{1}$ is $E T_{1}$-lifting and $M_{2}$ is $E T_{2}$-ifting , then $H_{1}=K_{1}+L_{1}$ and $H_{2}=K_{2}+L_{2}$, where $K_{1} \leq{ }_{\oplus} M_{1}$ and $L_{1} \ll E T M_{1}, K_{2}$ 
$\mathrm{S}_{\oplus} \mathrm{M}_{2}$ and $\mathrm{L}_{2} \ll_{E T} \mathrm{M}_{2}$. Then $\mathrm{H}=\mathrm{H}_{1} \oplus \mathrm{H}_{2}=\left(\mathrm{K}_{1}+\mathrm{L}_{1}\right) \oplus\left(\mathrm{K}_{2}+\mathrm{L}_{2}\right)=\left(\mathrm{K}_{1} \oplus \mathrm{K}_{2}\right)+\left(\mathrm{L}_{1} \oplus \mathrm{L}_{2}\right)$.to prove $\left(\mathrm{K}_{1} \oplus \mathrm{K}_{2}\right)$ is a direct summand of $M$. since $K_{1} \leq{ }_{\oplus} M_{1}$ then there exist $A_{1} \leq M_{1}$ and $A_{2} \leq M_{2}$ such that $M_{1}=K_{1}+A_{1}$ and $M_{2}=K_{2}+A_{2}$, then $M_{1}+M_{2}=\left(K_{1}+A_{1}\right)+$ $\left(K_{2}+A_{2}\right)=\left(K_{1}+K_{2}\right)+\left(A_{1}+A_{2}\right)$ and $\left(K_{1}+K_{2}\right) \cap\left(A_{1}+A_{2}\right)=0$, then $\left(K_{1}+K_{2}\right) \leq_{\oplus}\left(M_{1}+M 2\right)=M$ By Proposition (2.12) in [7], then( $\left.\mathrm{L}_{1} \oplus \mathrm{L}_{2}\right) \ll_{\mathrm{E}(\mathrm{T} 1 \oplus \mathrm{T} 2)} \mathrm{M}$. Thus $\mathrm{M}=\left(\mathrm{M}_{1} \oplus \mathrm{M}_{2}\right)$ is $\mathrm{E}\left(\mathrm{T}_{1} \oplus \mathrm{T}_{2}\right)$-lifting module.

Proposition 2.10:- Let $\mathrm{M}=\bigoplus_{i \in I} \mathrm{M}_{\mathrm{i}}$ be a" fully stable module" ; and $\mathrm{T}=\bigoplus_{i \in I} \mathrm{~T}_{\mathrm{i}}$, where $\mathrm{T}_{\mathrm{i}} \leq \mathrm{M}_{\mathrm{i}}$. If $\mathrm{M}_{\mathrm{i}}$ is $\mathrm{ET}_{\mathrm{i}}$-lifting, for each $i \in I$, then $M$ is a ET-lifting module .

Proof: Let $B$ be sub-module of $M$.For each $i \in I$, let $f_{i}: M \rightarrow M_{i}$ be the projection map. To prove $B=\bigoplus_{i \in I}\left(B \cap M_{i}\right)$. Let $\mathrm{y} \in \mathrm{B}$, then $\mathrm{y} \in \bigoplus_{i \in I} \mathrm{M}_{\mathrm{i}}$ and hence $\mathrm{y}=\sum_{i \in I} \quad y_{i}$, for $\mathrm{y}_{\mathrm{i}} \in \mathrm{M}_{i}$ and $\mathrm{y}_{\mathrm{i}} \neq 0$ for at most a finite number of $i \in \mathrm{I}$.Since $\mathrm{M}$ is fully stable, then $\pi_{i}(\mathrm{y}) \in \mathrm{B}$ and hence $\mathrm{f}_{i}(\mathrm{y}) \in \mathrm{B} \cap \mathrm{M} i$. Now $\mathrm{f}_{i}(\mathrm{y})=\pi_{i}\left(\sum_{i \in I} \quad y_{i}\right)=\mathrm{y}_{i}$. So $\mathrm{y}_{i} \in \mathrm{B} \cap \mathrm{M} i$ and hence $\mathrm{y}=\sum_{i \in I} \quad y_{i} \in \bigoplus_{i \in I}\left(\mathrm{~B} \cap \mathrm{M}_{\mathrm{i}}\right)$. Thus $\mathrm{B} \subseteq \bigoplus_{i \in I}\left(\mathrm{~B} \cap \mathrm{M}_{\mathrm{i}}\right)$. Thus $\mathrm{B}=\bigoplus_{i \in I}\left(\mathrm{~B} \cap \mathrm{M}_{\mathrm{i}}\right)$. Since $\mathrm{B} \cap \mathrm{M}_{\mathrm{i}} \subseteq \mathrm{M}_{\mathrm{i}}$ and $\mathrm{M}_{\mathrm{i}}$ is $\mathrm{ET}_{\mathrm{i}}$-lifting , then $\mathrm{B} \cap \mathrm{M}_{\mathrm{i}}=\mathrm{K}_{\mathrm{i}}+\mathrm{L}_{\mathrm{i}}$, where $\mathrm{K}_{\mathrm{i}} \leq_{\oplus} \mathrm{M}_{\mathrm{i}}$ and $\mathrm{L}_{\mathrm{i}} \ll<_{E \mathrm{Ei}} \mathrm{M}_{\mathrm{i}}$. Then $\bigoplus_{i \in I}\left(\mathrm{~B} \cap \mathrm{M}_{\mathrm{i}}\right)=\bigoplus_{i \in I}\left(\mathrm{~K}_{\mathrm{i}}+\mathrm{L}_{\mathrm{i}}\right)=\bigoplus_{i \in I} \mathrm{~K}_{\mathrm{i}}+\bigoplus_{i \in I} \mathrm{~L}_{\mathrm{i}}$. One can easily show that $\bigoplus_{i \in I} \mathrm{~K}_{\mathrm{i}} \leq \bigoplus_{i \in I} \mathrm{M}_{\mathrm{i}}$.By Proposition (2.13) in [7], then $\bigoplus_{i \in I} \mathrm{Li} \ll_{E \oplus_{i \in I} T i} \mathrm{M}$.ThenM is ET-lifting module.

\section{References}

[1] Fleury, p.,(1974),"Hollow Module and Local Endomorphism Rings" , Pac.J.Math., 53, 379-385.

[2] F. Kasch, Modules and Rings, Academic Press, Inc- London, 1982.

[3] X. Zhou and X. R. Zhang, "Small-Essential Submodules and Morita Duality", SoutheastAsian Bulletin of Mathematics, 35, 1051-1062, 2011

[4] R. Beyranvand and F. Moradi, "small submodule with respect to an arbitrary submodule", Journal of Algebra and Related Topcs Vol.3, No2, (2015),P43-51.

[5] I.M.A.Hadi, S.H.Aidi, "On e-Small Submodules", Ibn Al-Haitham Journal for Pure and Applied Science, 2015.

[6] K.R.Goodearl, "Ring Theory, Nonsingular Rings and Modules", Marcel Dekkel,1976.

[7] F.SH. Fandi and S.M. Yaseen," on essential (T-samll) submodule",Second international conference for applied and pure mathematics, 2019.

[8] R. Wisbauer, "Foundations of module and ring theory, Gordon and Breach", Philadelphia, 1991..

[9] V. Erdogdu, Distributive Modules, Can. Math. Ball 30(1987), (248-254).

[10] M. S. Abass, "on fully stable modules”, ph.D. Thesis, University of Baghdad, (1991). 